\title{
Accéder à un poste définitif de chercheur universitaire
}

\author{
Rapports à la carrière et articulations \\ avec la vie privée et familiale
}

\begin{abstract}
Bernard Fusulier ${ }^{1}$ et Hanna Pawelec ${ }^{2}$
[Résumé] À partir d'entretiens à caractère biographique de chercheurs ayant récemment obtenu un poste définitif au Fonds National de la Recherche Scientifique Belge, les auteurs analysent l'interférence entre le rapport à la carrière et la vie privée. Ils reconstruisent sur cette base trois figures-types de chercheur : le chercheur « exclusif » se focalisant entièrement sur le travail ; le chercheur « breadwinner » priorisant la carrière professionnelle sans pour autant sacrifier la vie de famille mais en déléguant en grande partie l'intendance domestique à sa conjointe; enfin le chercheur «équilibriste » qui tente de trouver un équilibre entre les engagements privés et professionnels. Ces deux dernières figurent sont fortement genrées, la première se conjuguant au masculin et la seconde au féminin. Cette analyse contribue avec d'autres à souligner combien l'articulation/interférence vie privée/vie professionnelle joue un rôle important dans le déploiement des carrières scientifiques. Si elle rappelle l'évidence qu'il est toujours plus difficile pour les femmes/mères que pour les hommes/pères de faire une carrière professionnelle, elle souligne aussi en creux que les configurations professionnelles qui permettent de naviguer avec succès dans le système reposent sur les personnes et pas sur l'organisation et l'institution.

Mots-clés : carrière scientifique, interférence travail-vie privée, genre, parentalité, origine sociale.

[Abstract] Drawn on biographical interviews with researchers having recently received a tenured position from the National Fund for Scientific Research (FNRS) in Belgium, the authors analyze the interference between attitudes toward professional career and private life. They reconstruct three ideal types of researchers: the "exclusive" researcher, focused entirely on scientific work; the "breadwinner" researcher, prioritizing a career without sacrificing family life by delegating household chores to his spouse; the "equilibrist" researcher, striving to find the work-life balance. The latter two figures are highly gendered, the first one being declined in masculine and the second one in feminine. This analysis contributes to highlight the extent to which the articulation of working life and private life plays an important role in the development of a scientific career. While reminding that it is still more difficult for women/mothers than for men/fathers to achieve a successful scientific career, this article emphasizes that successful professional configurations rely on individuals and not organizations.
\end{abstract}

${ }^{1}$ Université catholique de Louvain / FNRS.

${ }^{2}$ Université catholique de Louvain. 
Keywords: ccientific career, work-life interference, gender, parenthood, social origin.

\section{Introduction}

Faire carrière comme chercheur ${ }^{3}$ nécessite un engagement temporel et subjectif important qu'implique l'intégration dans un champ scientifique (Bourdieu, 1976) et une institution " gourmande » (" greedy institution »-Coser, 1977). La socialisation professionnelle des chercheurs conduit à l'apprentissage d'un « ethos » professionnel qui suppose la démonstration d'une vocation et un investissement entier dans la carrière professionnelle non guidé par un intérêt purement économique (Freidson, 1986). Le chercheur répondant à toutes les sollicitations, minorant les préoccupations domestiques, accédant à un poste stable selon un calendrier serré, apparaît comme un étalon auquel se comparent les chercheurs dans l'élaboration de leurs perspectives professionnelles (Dany, Louvel et Valette, 2011 ; Barbier, Fusulier, 2016). Cet étalon est peut-être d'autant plus prégnant depuis le déploiement d'une nouvelle régulation du travail scientifique autour d'une injonction politico-managériale à l'excellence, entendue notamment comme une mesure et une mise en équivalence de la productivité, de la compétitivité et de la mobilité des chercheurs, dans un contexte où de nombreux prétendants à une carrière scientifique et académique vivent une situation de précarité d'emploi (Ylijoki, 2010 ; Fusulier, del Rio Carral, 2012).

Bien que la population étudiante de l'enseignement universitaire ainsi que le groupe professionnel des enseignants-chercheurs se soient fortement féminisés cette dernière décennie (Le Feuvre, 2015), les carrières restent marquées par les phénomènes du « tuyau percé » (Alper, 1993 ; Meulders et al., 2012 ; Dubois-Shaik, Fusulier, 2015) et du « plafond de verre ( Marry, 2005; Fassa, Kradolfer, 2010) qui affectent les femmes. Les causes de ces inégalités ne relèvent plus tant d'une discrimination directe et explicite (lors des recrutements - Musselin, Pigeyre, 2008) que d'une organisation genrée (Acker, 1990) construite autour d'une figure masculine du chercheur comme "lonely hero» (Benschop, Brouns, 2003). Cette figure héroïque fait oublier que le rapport à la carrière se fabrique dans des dynamiques à la fois professionnelles et sociales (Avril et al., 2010) ainsi que familiales (Barbier, Fusulier, 2015). Plusieurs recherches montrent d'ailleurs qu'une cause des inégalités entre les sexes se loge dans l'articulation entre le privé et le professionnel (e.g Etzkowitz et al., 2000 ; Marry, Jonas, 2004 ; Case, Richley, 2013 ; del Rio Carral, Fusulier, 2013).

Voulant observer la façon dont la carrière se construit en relation avec la vie privée des chercheurs, nous avons interviewé trente-deux chercheurs qui ont été récemment nommés (depuis 2010 - voir encadré 1) par le Fonds National de la Recherche Scientifique (FNRS) en Belgique. Les chercheurs permanents du FNRS constituent un corps

\footnotetext{
${ }^{3}$ Nous utilisons le terme de chercheur au sens épicène, intégrant de ce fait tant les hommes que les femmes.
} 
spécifique du champ scientifique et académique belge, limités à quatre cent toutes disciplines confondues (dans les secteurs des sciences humaines et sociales - $\mathrm{SSH}$, celui des sciences et technologies - SST, et celui des sciences de la santé - SSS), pour l'ensemble des universités belges francophones (chaque université ayant elle-même un quota annuel qui lui est attribué). Ils sont sélectionnés sur dossiers par une commission scientifique $a d$ hoc à partir des critères les plus en phase avec l'excellence scientifique telle que définie aujourd'hui par l'institution (parcours académique, qualité du programme de recherche, dossier de publications, expériences internationales, crédits de recherche obtenus, lettres de recommandation, prix scientifiques...). Ces chercheurs doivent en outre être soutenus par l'université qui va les accueillir, et en dernière instance c'est celle-ci qui choisit les mandataires parmi la liste des chercheurs classés « excellents » par les commissions FNRS. Il est reconnu en Belgique que les chercheurs qualifiés FNRS sont ceux dont le «ticket d'entrée » dans un mandat permanent est le plus élevé, en tout cas au niveau du profil scientifique; une vingtaine en moyenne sont nommés chaque année, bien que leur nombre a chuté à dix et moins en 2015 et 2016 du fait des limites du quota qui implique qu'un mandat s'ouvre si un mandat se libère. Étudiant ces chercheurs qualifiés, notre objectif est de comprendre la façon dont ils ont vécu et organisé leur vie privée pour parvenir à décrocher ce statut. Par distance/proximité entre les récits et profils des chercheurs interviewés (voir l'encadré ci-dessous), nous avons ainsi reconstitué quelques types de rapport à la carrière et à la vie privée qui ont permis leur trajectoire "gagnante », dans le sens où elle a abouti à l'obtention d'un mandat définitif dans la recherche fondamentale. 
Échantillon et méthodologie de dégagement des idéaux-types

Notre échantillon est constitué de 16 femmes et 16 hommes ayant obtenu un poste permanent du FNRS entre 2010 et 2014 (sur une population totale de 92 individus recrutés durant cette période, dont 33 femmes), provenant de toutes les universités belges francophones et de tous les champs disciplinaires. Au moment de la nomination, les hommes de notre échantillon avaient entre 32 et 41 ans (34 ans en moyenne) et les femmes entre 32 et 45 ans (36 ans en moyenne). Ils sont majoritairement belges, à l'exception d'un homme et de quatre femmes ayant une nationalité étrangère. En ce qui concerne leur vie familiale, un homme et une femme sont célibataires. Sept personnes sont en couple avec un autre chercheur. Au moment de l'entretien, trois femmes et quatre hommes n'avaient pas d'enfant; avant leur nomination au poste permanent, 14 chercheurs n'étaient pas parents. La majorité des individus de notre échantillon provient d'un milieu social assez aisé : 26 d'entre eux ont au moins un parent avec des études supérieures (sur 29 dont nous avons des informations), parmi lesquels 23 ont au moins un parent avec un diplôme universitaire. II est notable que 14 d'entre eux travaillent dans le domaine proche de celui d'un ou des parents, et 13 ont été élevés par au moins un parent enseignant (à différents niveaux du système scolaire). Remarquons que les expériences de la mobilité internationale diffèrent selon le sexe des interviewés. Tous les hommes ont fait un séjour de longue durée (minimum 6 mois) alors que quatre femmes ne l'ont pas fait (elles provenaient toutes du secteur des sciences sociales et humaines). Les hommes étaient aussi plus nombreux à partir (au moins 6 mois) dans un pays anglophone (11 contre 4 femmes) et sur un autre continent (10 contre 5 femmes).

Les entretiens portaient principalement sur l'entrée au doctorat, le rapport à l'emploi, le rapport au travail et la vie privée. Chaque entretien a été synthétisé pour produire un « récit compréhensif » de quelques pages, permettant d'avoir une vision dynamique du parcours du chercheur (un enchaînement de ses situations de vie et de ses expériences vécues). Ensuite, nous avons procédé par une double opération qui tenait compte à la fois des profils socio-démographiques des chercheurs (sexe, situation privée, âge...) et des similitudes/différences entre les récits (e.g. la place que prenait leur engagement professionnel dans leur vie, leur situation conjugale et parentale, la manière dont ils concevaient et organisaient l'articulation travail/famille...) afin d'aboutir à des rapports à la carrière selon la méthode de l'idéal-type. Ainsi, le discours et le profil de certains chercheurs sont parfois très similaires, alors que d'autres tiennent un discours différent (au moins partiellement) et ont un autre profil. C'est en typifiant ces différences, c'est-à-dire en tentant de comprendre et de nommer la logique sous-jacente à l'expression convergente d'un groupe de chercheurs relativement à des expressions observables dans d'autres regroupements, que nous avons défini différentes figures de chercheur. Elles sont des idéaux-types dans le sens où elles relèvent d'une reconstruction épurée d'un certain rapport à la carrière ancré dans des biographies et conditions de vie. Si les trois figures de chercheur que nous avons dégagées (« exclusif », «breadwinner », « équilibriste ») sont loin d'être inattendues, elles éclairent sous une lumière particulière la façon dont se jouent les inégalités de genre dans les carrières scientifiques. 


\section{La figure du chercheur " exclusif " : focalisation sur la carrière dans un rapport exclusif au travail}

Dans ce premier cas de figure, il s'agit d'un chercheur entièrement investi dans son travail et considérant la recherche comme une activité peu compatible avec d'autres types d'engagement. Ses activités et engagements extra-professionnels sont alors réduits au minimum. Pour le chercheur « exclusif», sa disponibilité constitue une nécessité dans un environnement de plus en plus compétitif et productif. Près de la moitié des individus de notre échantillon se rapproche de cette première figure.

Pour un chercheur « exclusif », la recherche n'est pas un travail mais plutôt une passion ou même une manière de vivre, ce qu'illustrent les propos d'Alice et d'Alexandre.

C'est vrai que quelque part le cerveau n'est jamais au repos mais moi ça ne me dérange pas parce que moi c'est une passion donc c'est vrai que mes amis ils me traitent tous un peu des fois de « workaholic » mais j'ai envie de dire que c'est une passion, ce n'est pas un métier donc quelque part je vis ma passion et voilà. (Alice, nomination : 32 ans, en couple sans enfant, SST)

En fait, pour moi la recherche c'est pas du tout un travail, c'est ma vie. Comme je disais souvent et c'est toujours très vrai aujourd'hui : "Pour beaucoup de chercheurs, la recherche, ils travaillent pour y gagner leur vie ». Mais en fait, moi, c'est plutôt l'inverse, je gagne ma vie pour faire de la recherche. (Alexandre, nomination : 32 ans, en couple sans enfant, SSH)

Sans pour autant ignorer que certains chercheurs peuvent surjouer leur dévouement à la recherche (Beaufays et Krais, 2005), celle-ci étant une passion, le chercheur « exclusif » y consacre quasi tout son temps, bien au-delà des heures contractuelles (norme légale des 38 heures par semaine). Les moments de surinvestissement accompagnent souvent les périodes précédant des échéances importantes, fin d'un projet ou d'un mandat. Il arrivait ainsi à Camille (nomination : 32 ans, en couple sans enfant, SSH) de travailler 18 heures par jour. Pénélope (nomination : 40 ans, en couple sans enfant, SSS) mentionne une période où avec son équipe « pendant six mois on travaillait de 7 heures du matin jusqu'à 2 heures du matin et 7 jours par semaine ».

Un chercheur « exclusif » travaille le soir, la nuit et le week-end. Dans ce choix, il est guidé non seulement par l'intérêt pour sa recherche mais aussi par l'instabilité de sa position. Derrière cet investissement, nous percevons la figure idéale d'un chercheur répondant à toutes les exigences du monde de la recherche, et pour lequel les critères d'excellence, tels que le nombre de publications et des séjours de longue durée à l'étranger, favorisent un engagement qui ne souffre pas d'une implication annexe, notamment dans une vie familiale.

Le chercheur modèle est sans doute celui qui est tout voué à sa carrière, à son institution, à... qui, qui peut être disponible à toute heure, qui... répond vite à ses courriels, qui est hyper investi. (Camille, nomination : 32 ans, en couple sans enfant, $\mathrm{SSH}$ ) 
Pour être disponible à tout moment et poursuivre la recherche, le chercheur limite ses jours de congé au minimum. Même pendant les vacances, Muriel lit des articles et Manuel en rédige au bord de la piscine. Se détacher du travail est vécu comme difficile, ce dont témoignent Thomas et Lola repris ci-après.

Je me déconnecte pas vraiment parce qu'il y a souvent une chose ou l'autre en cours [...] Ça m'arrive de revenir ici pendant mes vacances pour préparer une réunion ou autre. (Thomas, nomination : 35 ans, en couple sans enfants, SST) On peut travailler le week-end, on peut travailler les vacances, on a... j'ai même du mal à me dire «non je prends des vrais congés ou je n'ouvre pas mon ordinateur » j'ai... c'est un petit peu devenu difficile. (Lola, nomination : 34 ans en couple sans enfant, SSH)

Plusieurs témoignages laissent apparaitre que la vie de couple est compliquée à stabiliser en raison de la pression temporelle et de la flexibilité exigée par l'activité scientifique et académique. Pour certains chercheurs, le fait d'être célibataire est alors vécu comme un avantage par rapport à leurs pairs, car ils peuvent davantage adapter leur vie aux exigences du travail, faire des déplacements à l'étranger quand ils le veulent et réaliser des séjours longs dans d'autres laboratoires...

Moi j'ai toujours habité toute seule, j'ai toujours voyagé, déménagé, c'était toujours facile, j'avais un horaire avant de 10 heures au labo, 12 heures [...] pendant mon PhD quand j'ai commencé à avoir pas mal de soucis pour avoir mes résultats, c'était 12/14h par jour toute la semaine (Muriel, nomination : 43 ans, en couple, enfant de 4 ans, SST)

Certains choisissent de vivre seul délibérément. C'est le cas de Mathieu dont une première relation conjugale n'a pas survécu à l'absence engendrée par un séjour postdoctoral à l'étranger. Bien qu'il se soit ensuite remis en couple, il va à nouveau rompre cette relation pour repartir dans un autre pays quelques mois plus tard. Finalement, il décide de rester célibataire jusqu'à son retour définitif en Belgique. Le couple n'est pas toujours un obstacle à un investissement fort dans le travail. Lola mentionne une période où elle était en couple avec un " fou de travail ", alors qu'Alice et son mari vivent séparément pour le bien de leurs carrières respectives. En revanche, pour ce type de chercheur, avoir des enfants semble incompatible avec un investissement total. Lola parle de l'« autocensure » des femmes par rapport au projet familial, mais le cas de Pierre illustre qu'il s'agit d'un phénomène présent également chez les hommes.

En tout cas de le reporter à un moment où on se dit que la carrière sera plus stable... que ce sera plus... Faire un enfant quand on est post-doctorat, bah c'est peut-être moins bien vu parce que... parce qu'il est plus difficile à gérer et à porter parce qu'on se dit « ah bah il va falloir que je parte faire un séjour à l'étranger [...] il y une sorte de [...] autocensure (Lola, nomination : 34 ans en couple sans enfant, SSH) 
Pour me marier, pour avoir un enfant j'ai même attendu d'avoir un poste permanent. [...] il faut vraiment que mon CV soit le meilleur entre mes compétiteurs. Pour achever ça il faut travailler jour et nuit. Si je travaille jour et nuit je ne peux pas être un père présent. Ma femme a aussi sa carrière à elle. Je ne trouve pas juste que quelqu'un doit sacrifier sa carrière. (Pierre, nomination : 35 ans, en couple sans enfant, SST)

\section{La figure du chercheur " breadwinner » : mener une carrière professionnelle tout en ayant un engagement familial, avec un rapport prioritaire au travail et une délégation des charges domestiques}

Un rapport prioritaire au travail caractérise ce type de chercheur, très investi dans son travail mais qui accorde parallèlement une réelle importance à leur vie familiale. Il a été surtout repéré à travers le discours de pères constituant un quart de notre échantillon. Il renvoie au modèle traditionnel de la division sexuelle de travail avec un homme fortement engagé dans sa vie professionnelle, assurant un rôle de « gagne-pain » (d'où la qualification de «breadwinner ») et de référence statutaire pour sa famille, et une conjointe qui prend largement en charge l'intendance domestique. Grâce à ce soutien, il peut adapter ses horaires aux activités professionnelles sans devoir trop se préoccuper des obligations relevant de la vie familiale. Néanmoins, il doit chercher des arrangements avec sa compagne.

Ainsi, le chercheur peut être disponible pour son travail, partir à un colloque quelques jours, faire un séjour à l'étranger, assister à des réunions tardives... Toutefois, l'agenda est le résultat d'une négociation conjugale qui prend appui sur une asymétrie des investissements professionnels et familiaux, et donc des positions sociales.

Ça fait c'est très machiste de dire ça, mais parce que mon épouse est enseignante et elle a pu prendre plus facilement du temps [...] la garde des enfants n'a jamais été un problème [...] comme chercheur le problème se serait posé d'une manière beaucoup plus importante. (Gérard, nomination: 32 ans, en couple avec 3 enfants de 7, 5 et 2 ans, SSH)

Disons que c'est relativement équitable [...] Mais je crois qu'au final par défaut je crois qu'elle va faire plus en fait, et c'est juste que par défaut je vais faire moins, je vais avoir tendance à me sentir plus stressé [...] par mon boulot c'est con parce que en fait normalement c'est un boulot... [...] du coup je crois qu'elle prend aussi beaucoup sur elle pour gérer ça. (Gilles, nomination : 36 ans, en couple avec 1 enfant de 2 ans, SST)

Remarquons que le modèle peut être inversé au plan du sexe, avec une femme fortement engagée dans sa carrière scientifique et soutenue par un conjoint qui porte la responsabilité des tâches familiales. 
Contrairement au chercheur « exclusif », le chercheur «breadwinner » n'est pas libéré des contraintes familiales et en tient compte. Par exemple, la mobilité internationale de longue durée n'est pas impossible, mais fait l'objet d'un accord entre les conjoints sur le type de mobilité envisagée (une expérience d'expatriation de la cellule familiale, des déplacements alternants...) comme l'illustre Jean ci-dessous.

C'était un peu compliqué parce que j'avais déjà des enfants, donc ma femme restait à X [domicile] et donc j'allais deux trois jours par semaines [à l'étranger] [...] mais c'était un an, et c'était le sacrifice, on savait bien que si je ne faisais pas ça je n'aurais jamais de poste fixe quoi. (Jean, nomination: 36 ans, en couple avec un enfant de 3 ans, SST)

Cette solution semble acceptable et gérable si elle est bien limitée dans le temps. Mais oui, ça était mon épouse qui a accepté de, de que je parte plus de temps, deux fois six mois et donc qui a accepté de s'occuper des enfants [...] à partir du moment que c'était temporaire, ça va. (Gérard, nomination: 32 ans, en couple avec 3 enfants de 7, 5 et 2 ans, SSH)

$\mathrm{Au}$ moment où le chercheur décide de partir quelques mois, la prise en charge pratique des enfants repose sur sa conjointe. La disponibilité de cette dernière est donc fondamentale, c'est pourquoi la réduction de son temps de travail professionnel constitue une mesure privilégiée. Un environnement soutenant s'avère aussi souvent nécessaire, comprenant notamment l'accès à une crèche pas trop éloignée du domicile ou l'aide intergénérationnelle au sein de la famille (l'importance de l'aide des grands-parents des enfants a été évoquée à plusieurs reprises).

Malgré un investissement élevé dans son travail, le chercheur «breadwinner» valorise le temps passé avec sa conjointe et ses enfants. Il accepte à certaines occasions d'inverser l'ordre de ses priorités et de privilégier ses obligations familiales, profitant d'ailleurs de l'autonomie que lui octroie le métier de chercheur: travailler à la maison lorsque l'enfant est malade, annuler une conférence quand la situation familiale le demande... Cependant, lorsqu'une urgence professionnelle se manifeste, il n'hésite pas à donner la priorité au travail.

Je rentre généralement vers 17 heures [...] vers 20 heures 30 je recommence à travailler. Bon ça c'est, entre guillemets, le quotidien quand il y a rien d'autre. Maintenant, i l y a très souvent des réunions qui se mettent ou quand on est en voyage évidemment là tout est chamboulé. (Gaétan, nomination : 34 ans, en couple avec 3 enfants de 5, 3 et 1 ans, SSH)

Autrement dit, soutenu par son épouse, le chercheur ne limite que rarement son investissement professionnel mais il peut l'ajuster aux besoins familiaux.

Dans la gestion quotidienne de cette vie et notamment le choix aussi de périodes de voyages que je peux choisir, notamment bon les jours aux archives, je fais une semaine avant ou après, ça c'est une chose qu'il faut discuter avec mon épouse. (Gaétan, père de 3 enfants, SSH) 
Soulignons que plusieurs chercheurs se rapprochant de ce type estiment être désavantagés dans la concurrence face aux chercheurs célibataires et plus étonnement face aux chercheures-mères ${ }^{4}$ qui, disent-ils en substance, sont reconnues dans leur maternité au point qu'une grossesse peut étendre leur droit à postuler plus tardivement à un mandat définitif du FNRS5. Il y a donc un sentiment que la paternité n'est pas reconnue par l'institution.

\section{La figure du chercheur " équilibriste » : combiner carrière et famille en tentant d'équilibrer les deux par des arrangements permanents}

Dans ce cas de figure, nous avons affaire à des chercheurs qui tentent de symétriser leurs implications professionnelles et familiales. Contrairement au type précédent, cette quête d'équilibre est principalement évoquée par des chercheures-mères. Certes, elles peuvent aussi bénéficier d'un support conjugal dans leur carrière, qui est d'ailleurs surtout présent lors de moments particuliers (séjours à l'étranger, des échéances imminentes, fins des contrats...) mais, au quotidien, elles sont en première ligne pour gérer les obligations familiales. Pour y parvenir, la chercheure « équilibriste » rationalise son temps, avec des journées très structurées qui, en général, ne lui permettent pas d'assister aux réunions placées en soirée ou à la foule d'activités, formelles et informelles, qui se déroule dans l'institution académique en dehors des horaires classiques de travail (voir extrait ci-dessous). Les chercheures interviewées, qui nous ont permis de dégager cette figure de l'« équilibriste », constituent le dernier quart de notre échantillon.

Je me disais, j'ai envie d'assister aux conversations où tout le monde se voit en fin de journée et moi je devais partir quoi. Je suis très stricte sur mes horaires, je quitte à 5 heures parce que c'est mon équilibre aussi, j'ai envie de rentrer à la maison, cuisiner, m'occuper des enfants. Si j'ai eu des enfants, c'est pour les voir. (Cassandra, nomination: 35 ans, en couple avec 3 enfants de 10, 8 et 4 ans, SST)

Marine (nomination : 33 ans, en couple avec 2 enfants de 4 et 3 ans, SSH) souligne aussi sa bonne gestion du temps et sa sélectivité par rapport aux réunions et d'autres engagements. Simultanément, le fait d'avoir des enfants semble jouer positivement sur la qualité du travail, en permettant des moments de coupure et de déconnexion salutaires pour un meilleur équilibre personnel, comme l'exprime aussi Manon ci-dessous.

4 Suivant une tendance actuelle, nous féminisons le mot chercheur en chercheure plutôt que d'utiliser la forme féminine traditionnelle «chercheuse », car le suffixe « euse » peut être senti comme dévalorisant (voir Lenoble-Pinson, 2006).

5 Le FNRS octroie en effet une augmentation d'une année par maternité l'ancienneté maximale entre l'obtention du titre de docteur et la candidature à un mandat de chercheur qualifié. 
Je pense que je suis plus efficace quand j'ai une deadline et que je sais qu'après je vais m'occuper des enfants [...] Pour moi, c'est indispensable, je n'envisagerais pas de faire la même chose en étant sans vie de famille, ça me permet de déconnecter. (Manon, nomination : 33 ans, en couple avec 2 enfants de 3 et 6 ans, SST)

Toutefois, cette structuration n'empêche pas le sentiment d'avoir « une double journée de travail », laquelle engendre une grande fatigue. Entre la vie de chercheure et la vie familiale, il n'y a pas beaucoup de répit.

Je pense que s'il y en a une qui a souffert c'est moi, parce que c'est fatigant, difficile d'être présente partout et d'essayer de faire bien partout. Et il y a des moments où j'ai cru comprendre ce que voulait dire un « burn out » mais aussi parce que j'ai les deux à gérer et que j'ai envie de faire les deux bien. Mais ma vie familiale et ma vie de couple, non. Je ne peux pas dire qu'on en ait souffert. (Adeline, nomination : 45 ans, en couple avec 2 enfants de 16 et 9 ans, SSS)

Si une bonne structuration du temps est une condition pour parvenir à combiner les deux milieux de vie, elle ne suffit pas car il existe par exemple un hiatus entre les horaires professionnels et les horaires scolaires ou des crèches. Il importe alors de trouver d'autres solutions. Ainsi, Chloé a déménagé plus près de sa famille pour pouvoir compter sur l'aide de ses propres parents ; Marine, dont le mari a un poste à haute responsabilité professionnelle, a trouvé un arrangement avec une voisine et sa belle-mère.

On habite assez loin de notre famille mais on a une voisine qui est à la retraite et qui vient souvent les chercher de l'école [...] Et ma belle-mère vient tous les mercredis chez nous parce que les mercredi après-midi il n'y a d'école. (Marine, nomination : 33 ans, en couple avec 2 enfants de 4 et 3 ans, SSH)

À côté de l'organisation du quotidien, la chercheure « équilibriste » se doit aussi de gérer les déplacements et séjours professionnels à l'étranger en tenant compte des impératifs familiaux, et encore plus en anticipant la manière dont ils vont pouvoir être assurés comme en témoigne Manon ci-dessous :

Quand je fais des missions à l'étranger, si je n'avais pas cela [soutien du conjoint], ce serait impossible. Je m'organise, quand je le sais suffisamment à l'avance, pour qu'il y ait l'un ou l'autre trajet qui soit assuré par d'autres personnes, pour que tout ne soit pas sur la tête de mon mari (Manon, nomination : 33 ans, en couple avec 2 enfants de 3 et 6 ans, SST)

Malgré le soutien conjugal, les déplacements à l'étranger peuvent déstabiliser l'équilibre et produire un mal-être : le mari qui se plaint, la culpabilité à distance si l'enfant tombe justement malade, le sentiment que l'enfant est affecté par l'absence de la mère... autant de propos entendus. Bref, la chercheure est amenée à rapidement restaurer des routines apaisantes pour elle-même et son entourage. 
Dans le cas de séjours de longue durée, qui est l'un des critères pris en considération pour obtenir un mandat définitif, il s'agit alors de le réaliser avec le conjoint et les enfants. L'expérience internationale, pour maintenir l'équilibre recherché, doit être définie comme un projet de couple.

Donc c'était le bon plan parce qu'il [son mari] savait quand même me suivre [pour des raisons professionnelles]. II fallait savoir où partir. Et donc là, pour être honnête, c'est plus ou moins lui qui a choisi. [...] On ne l'a vraiment pas regretté, ni pour la recherche ni pour la vie là-bas. (Cassandra, nomination : 35 ans, en couple avec 3 enfants de 10, 8 et 4 ans, SST)

Il en est de même pour Manon.

Il y a aussi le fait que l'employeur de mon mari a accepté son détachement. [...] je ne serais pas partie seule sans voir mon enfant pendant 1 an et demi. [...] Cela n'aurait pas été envisageable pour moi. Là, j'avais dit « je ne pars pas et je dois me réorienter » (Manon, nomination : 33 ans, en couple avec 2 enfants de 3 et 6 ans, SST)

Cette difficile séparation renvoie à la construction sociale de la maternité supposant un besoin particulier du soutien maternel pour le développement de l'enfant (Pfau-Effinger, 1998). Elle est très présente dans le discours des mères interviewées et peut rendre impossible une expérience internationale conséquente. C'est le cas de Chloé qui parle de « manque » en référence à un séjour long à l'étranger, même s'il ne l'a pas empêchée d'être finalement nommée car elle a pu faire valoir d'autres atouts dans son CV. Par exemple, ce qui m'a manqué, c'est un séjour de longue durée à l'étranger qui est devenu maintenant un critère important. Je ne l'ai pas fait parce que c'est arrivé à l'époque où j'ai eu mes enfants et que je me voyais mal partir etc. (Chloé, nomination : 37 ans, en couple, 2 enfants de 7 et 5 ans, SSH)

En somme, la chercheure qui combine sa carrière et sa vie de famille est dans une situation d'équilibriste qui marche sur un fil. Comme cette image le laisse entrevoir, l'équilibre met aussi en jeu le corps ; des aléas de santé ou la grossesse par exemple ne sont sans effets sur l'exercice de funambulisme.

\section{Conclusion}

L'analyse des entretiens de chercheurs ayant obtenu un poste définitif dans le champ scientifique belge francophone laisse entrevoir une réalité plurielle qui n'est pas pour autant infinie. En effet, trois types de conduite de trajectoire ont été identifiés autour de la façon dont les chercheurs investissent leur travail professionnel et envisagent ou pratiquent son articulation avec leur vie privée (et familiale en particulier).

Dans le premier type (la figure du chercheur « exclusif »), nous avons un chercheur, homme ou femme, dont la vie professionnelle et la carrière constituent l'épine dorsale de sa vie. Il ne se laisse pas divertir par d'autres engagements afin de mettre toute les chances de son côté pour accéder à un poste définitif. Le célibat et, surtout, l'absence 
d'enfant semblent propices à cette logique de carrière. Observons également, sans pouvoir généraliser le constat, que c'est aussi à l'intérieur de ce type que nous retrouvons les quelques chercheurs provenant d'un milieu social que l'on peut qualifier de populaire eu égard aux diplômes et catégories socio-professionnelles des parents. Dans une enquête ultérieure par questionnaire auprès de l'ensemble des chercheurs permanents du FNRS, une corrélation entre l'origine sociale et la situation conjugale/parentale a d'ailleurs pu être observée (Pawelec, 2016). Sans doute que pour un chercheur d'origine populaire, il importe de maximiser son investissement professionnel et de minimiser d'autres engagements sociaux pour passer les épreuves de la carrière universitaire et parvenir à décrocher le mandat de chercheur qualifié du FNRS convoité en Belgique francophone. Peut-être avons-nous aussi affaire à un mécanisme sociologique identifié par Pierre Bourdieu et Monique De Saint Martin qui, à la manière des « oblats » qu’ils étudient dans le champ épiscopal, amène ces chercheurs à être disposés à « tout donner à une institution qui leur a tout donné » (Bourdieu, De Saint Martin, 1982 : 5).

Notre étude nous permet de repérer deux autres types qui expriment deux autres figures de chercheur, l'une qui se conjugue au masculin (le chercheur « breadwinner »), l'autre au féminin (la chercheure « équilibriste »).

Dans le cas de la figure du chercheur «breadwinner », nous avons affaire tendanciellement à des pères (même si nous ne devons pas exclure des mères à travers une inversion des rôles de genre) qui donnent priorité à leur carrière professionnelle, sans pour autant sacrifier leur vie de famille ${ }^{6}$. Toutefois, cette dernière est plutôt ajustée à leur implication scientifique, avec une conjointe qui les décharge grandement du quotidien de l'intendance domestique et des soins aux enfants. Il s'agit d'un schéma classique dans la division sexuelle du travail productif et du travail reproductif. Toutefois, peutêtre n'est-il pas la pure reproduction du modèle traditionnel d'un ordre genré naturalisé (donc non discuté) car l'enjeu de la négociation conjugale paraît important dans la mesure où il s'agit de trouver un compromis entre les partenaires malgré l'asymétrie dans l'affectation sexuée des tâches domestiques et de l'investissement professionnel.

La troisième figure est celle de la chercheure « équilibriste », surtout représentée par des chercheures-mères (même si nous ne devons pas exclure que certains pères puissent s'en rapprocher). Elles tentent de garder un équilibre entre leurs engagements professionnels et leurs engagements familiaux, et principalement parentaux. Ce cas de figure est certainement le plus complexe, mais pas nécessairement le plus improbable étant donné la féminisation progressive des carrières scientifiques (en Belgique, 40 \% des nouveaux proclamés au titre de docteur sont de sexe féminin). Certes, en ayant étudié des trajectoires "gagnantes", nous avons collecté les témoignages de femmes qui sont parvenues à maintenir ce relatif équilibre, une «work/life balance». La question qui se pose est de savoir si le phénomène de déperdition des femmes dans la carrière

${ }^{6}$ L'analyse des « pères gagnants » n'est évidemment pas neuve et déjà bien commentée par de Singly (1996) ou Gadéa et Marry (2000) mais trouve ici une expression spécifique. 
ne relèverait pas de l'impossibilité pour nombre d'entre elles de construire un tel équilibre dans la durée, notamment parce qu'elles ne bénéficieraient pas des supports privés qui leur paraissent indispensables.

À cet égard, les travaux antérieurs auprès de chercheurs en postdoctorat du FNRS (les chargés de recherches - emploi à durée déterminée : Fusulier, del rio Carral, 2012 ; del Rio Carral, Fusulier, 2013 ; Barbier, Fusulier, 2015) soulignaient déjà l’importance des supports configurationnels que les chercheurs trouvent (ou non) à la fois dans leur milieu professionnel (un promoteur soutenant, l'accès à un réseau porteur, un article bien publié, des collègues bienveillants...) et dans leur milieu privé (faible contrainte conjugale ou familiale, ou support fort des parents et conjoint-e-s, accès aisé à des services, localisation à proximité du lieu de travail...). Or ces deux types de supports ne semblent pas également répartis entre les sexes, et des configurations de soutien déficitaires affectent tendanciellement davantage les femmes et les mères.

Par ailleurs, si on constate que les configurations qui permettent de naviguer avec succès dans le système reposent principalement sur les personnes et leur situation de vie, on perçoit peu les supports organisationnels. Pourtant, il existe une prise de conscience des responsables des grandes institutions scientifiques. Par exemple, la Charte européenne des chercheurs intègre la proposition suivante : les employeurs et bailleurs de fonds « devraient viser à fournir des conditions de travail qui permettent aux chercheurs tant féminins que masculins de combiner la famille et le travail, les enfants et la carrière ${ }^{7}$ ». Au niveau des universités, des mesures ont été récemment prises, comme l'illustre la Charte pour l'équilibre des temps de vie signée en septembre 2015 par l'École des hautes études en sciences sociales de Paris. Mais simultanément, les institutions scientifiques mettent en place des outils d'évaluation qui relèvent d'un régime comptable-productiviste court-termiste (dans un laps de temps court, il s'agit de publier plus, être plus mobile, obtenir plus de financements de recherche, de rendre des comptes constamment...[Fusulier, 2016]) qui par effet de conséquence renforce la pression sur les chercheurs et accentue la tension vie professionnelle/vie privée toujours plus fortement vécue par les femmes que par les hommes. Il y a donc lieu de questionner la régulation même de l'espace scientifique, mais ceci ouvre un autre débat.

\section{Bibliographie}

ACKER J. (1990), « Hierarchies, jobs, and bodies: A theory of gendered organizations », Gender e Society, vol. 4, n 2, p. 139-58.

AlPER J. (1993), « The pipeline is leaking women all the way along », Science, vol. 260, $\mathrm{n}^{\circ}$ 5106, p. 409-411.

Avril C., Cartier M., Serre D. (2010), Enquêter le travail, Paris, La Découverte.

\footnotetext{
${ }^{7}$ http://ec.europa.eu/euraxess/pdf/brochure_rights/eur_21620_en-fr.pdf.
} 
BARBIER P., FUSULIER B. (2015), « L'interférence parentalité-travail chez les chercheur-e-s en post-doctorat: le cas des chargé-e-s de recherches du Fonds National de la Recherche Scientifique en Belgique », Sociologie et Sociétés, vol. 47, nº 1, p. 225-248.

BARBIER P., FUSULIER B. (2016), « Comprendre l'expérience de la carrière scientifique et les inégalités entre les sexes au regard de l'interférence Travail/Famille », in R. ROGERS, P. MOULINIER (dir.), Les femmes dans le monde académique, Rennes, Presses Universitaires de Rennes, p. 81-94.

BeAUFAYS S., KRAIS B. (2005), « Femmes dans les carrières scientifiques en Allemagne: les mécanismes cachés du pouvoir », Travail Genre et Société, vol. 14, n² 2, p. 49-68.

Benschop Y., Brouns M. (2003), « Crumbling Ivory Towers: Academic Organizing and its Gender Effects », Gender Work and Organization, vol. 10, n 2, p. 194-212.

BOURDiEu P. (1976), « Le champ scientifique», Actes de la recherche en sciences sociales, vol. 2, nº 2-3, p. 88-104.

BouRdieu P., De Saint Martin M. (1982), « La sainte famille. L'épiscopat français dans le champ du pouvoir », Actes de la recherche en sciences sociales, vol. 44, $\mathrm{n}^{\circ}$ 1, p. 2-53.

CASE S. S., RICHLEY B. A. (2013), « Gendered institutional research cultures in science: the post-doc transition for women scientists », Community, Work e Family, vol. 16, $\mathrm{n}^{\circ} 3$, p. 327-349.

COSER L. (1974), Greedy Institutions, New York, The Free Press.

Dany F., Louvel S., Valette A. (2011), « Academic Careers: The limits of the "boundaryless approach" and the power of promotion scripts », Human Relations, vol. 64, $\mathrm{n}^{\circ} 7$, p. 971-996.

Del Rio CaRRAL M., Fusulier B. (2013), « Jeunes chercheurs face aux exigences de disponibilité temporelle», Temporalités: Revue de sciences humaines et sociales, vol.18. URL: https://temporalites.revues.org/2614.

De Singly F (1996), Le soi, le couple, la famille, Paris, Nathan.

Dubois-Shaik F., Fusulier B. (2015), « Academic Careers and Gender Inequality: Leaky Pipeline and Interrelated Phenomena in Seven European Countries », GARCIA working papers, vol. 5, University of Trento. URL : http://garciaproject.eu/wp-content/uploads/2015/11/GARCIA_report_wp5D.pdf.

EtzKowitz H., Kemelgor C., Uzzi B. (2000), Athena Unbound: the Advancement of Women in Science and Technology, Cambridge, MA, Cambridge University Press.

FASSA F., KRADOlfer S. (coord.) (2010), Le plafond de fer de l'université. Femmes et carrières, Zurich, Seismo.

FREIDSON E. (1986), « Les professions artistiques comme défi à l'analyse sociologique », Revue Française de sociologie, vol. 27, nº 3, p. 431-443. 
Fusulier B., Del Rio Carral M. (2012), Chercheur-e-s sous haute tension!, Louvain-laNeuve, Presses universitaires de Louvain.

FUSULIER B. (2016), « Faire une carrière scientifique aujourd'hui. Quelques clés de lectures et critiques », in E. Zaccai, B. Timmermans, M. Hudon, B. ClerbauX, B. LeClercQ, H. BERSini (dir.), L'évaluation de la recherche en question(s), Bruxelles, Académie Royale de Belgique, p. 101-110.

GADÉA C., MARRY C. (2000), « Les pères qui gagnent: descendance et réussite professionnelle des ingénieurs », Travail, Genre et Sociétés, vol. 3, p. 109-135.

Le Feuvre N. (2015), « Contextualizing Women’s Academic Careers in Cross-National Perspective », GARCIA working papers, vol. 3, University of Trento. URL : http://garciaproject.eu/wp-content/uploads/2015/10/GARCIA_report_wp3.pdf.

LENOBLE-Pinson M. (2006), « Chercheuse ? Chercheur ? Chercheure ? Mettre au féminin les noms de métier et les titres de fonction ", Revue belge de philologie et d'histoire, vol. 84, n 3, p. 637-652.

MARry C. (dir.) (2005), "Enquête sur les promotions CR-DR dans une section des sciences de la vie du CNRS », Rapport de recherche pour la Direction générale du CNRS et la Mission pour la place des femmes au CNRS, Juin.

MARRY C., JONAS I., (2005), « Chercheuses entre deux passions », Travail, genre et sociétés, vol. $14, n^{\circ} 2$, p. 69-88.

Meulders D., O’Dorchai S., Simeu N. (2012), Les inégalités entre femmes et hommes dans les universités francophones de Belgique, rapport de recherche GENIUF, Bruxelles, Université Libre de Bruxelles.

PAWELEC H. (2016), La carrière scientifique à l'épreuve du genre et de l'origine sociale, mémoire de Master 120 en sociologie, prom. B. FusULIER, Louvain-la-Neuve, Université catholique de Louvain.

PFAU-EFFInGER B. (1998), «Gender cultures and the gender arrangement - a theoretical framework for crossnational comparisons on gender », Innovation: the European Journal of Social Sciences, vol. 11, $\mathrm{n}^{\circ}$ 2, p.147-166.

Rossiter M. (1993), « The Matthew Matilda Effect in Science», Social Studies of Science, vol. 23, n 2, p. 325-341.

YLIJOKI O. H. (2010), « Future orientations in episodic labour: Short-term academics as a case in point », Time er Society, vol. 19, n 3, p. 365-86. 
